\title{
Run-time Power Control Scheme Using Software Feedback Loop for Low-Power Real-time Applications
}

\author{
Seongsoo Lee \\ Takayasu Sakurai \\ Center for Collaborative Research and Institute of Industrial Science, University of Tokyo \\ 7-22-1 Roppongi, Minato-ku, Tokyo, Japan, 106-8558 \\ Tel: +81-3-3402-6226 \\ Fax: +81-3-3402-6227 \\ e-mail: \{cupid, tsakurai\}@ iis.u-tokyo.ac.jp
}

\begin{abstract}
A novel hardware-software cooperative power control scheme, namely a software feedback loop, is proposed to lower power consumption of VLSI systems. The proposed runtime voltage and frequency control scheme guarantees the realtime execution of applications. It avoids interface problems and also provides "binary-code compatibility". Using a software analysis environment, the power control scheme is shown to achieve more than $90 \%$ power reduction for real-time MPEG-4 SP@L1 video encoding, taking into consideration the transition delay between voltage and frequency levels.
\end{abstract}

\section{INTRODUCTION}

Over the past several years, reduction of power consumption is taking on significant importance in VLSI system design, especially for portable, battery-powered devices such as a digital cellular phone and a personal digital assistant (PDA).

One promising approach in power reduction is dynamic voltage scaling [1][2]. In the scheme, supply voltage is reduced to the lowest possible level to achieve the lowest power consumption, since the power consumption of CMOS circuits is proportional to the square of the supply voltage. As the supply voltage decreases, the speed of CMOS circuits also decreases. Therefore, the supply voltage should be dynamically controlled based on the workload variation.

Recently, extensive studies have been carried out on the dynamic voltage scaling [1]-[5], based on compile-time supply voltage scheduling. These approaches, however, are not suitable for real-time applications, because they do not guarantee for the program to be finished in a given time interval. Neither, it does provide the optimum supply voltage, because the workload variation of each task is not exactly known at compile-time and it is data dependent.

Moreover, in the previously published approaches, system clock frequency can have arbitrary values, which may cause interface problems to exchange data with external memories and peripheral LSI's. Especially, this interface problem becomes serious for external devices such as a cathode-ray tube (CRT), a liquid-crystal display (LCD), and a radio frequency (RF) front-end. Usually, these devices operate at a constant clock frequency so that complicated interface circuits may be needed.

A dynamic voltage scaling scheme often employs a DCDC converter and a frequency synthesizer to control the supply voltage and clock frequency. DC-DC converters have rather slow transition delay compared with processor cycle time. The transition delay should be also taken into account, but most of the conventional approaches do not.

Voltage-frequency relationship is important in dynamic control of the supply voltage. In the conventional approaches, the relationship is stored in the form of dedicated hardware and/or customized software. The voltage-frequency relationship, however, depends strongly on the process technology and architecture, which means that hardware redesign or software recompilation is required when the generation of a processor changes.

In this paper, a novel hardware-software cooperative scheme is proposed to lower power consumption of VLSI systems. It consists of (1) a simple power control chip with an on-chip DC-DC converter and a frequency generator, (2) a simple run-time power control algorithm using software feedback loop, and (3) a device driver for considering 
voltage-frequency relationship of a target processor.

The dynamic software feedback scheme proposed in the paper guarantees real-time execution of the application for the first time and achieves more power saving over compiletime supply voltage scheduling. It is because the supply voltage is controlled adaptively in run-time and the scheme takes the transition delay into account. The power control chip generates $\mathrm{f}_{\mathrm{CLK}}, \mathrm{f}_{\mathrm{CLK}} / 2, \mathrm{f}_{\mathrm{CLK}} / 3, \ldots$ to avoid interface problems, where $f_{C L K}$ is the master clock frequency. Voltage-frequency relationship is given in the form of device driver program being separate from the application program. Therefore, neither recompilation nor reprogramming of the application program is needed even when a processor is upgraded.

Since the power control chip is separate from the processor, proposed scheme can be directly applied to many existing commercial processor systems.

In order to show the effectiveness of the scheme, performance evaluation is conducted using Pentium II code set and MPEG-4 video encoding [6], which is going to be the killer application for portable systems that require real-time execution.

\section{ARCHITECTURE}

The outline of the proposed run-time power control scheme is shown in Fig. 1. The power control algorithm is embedded in a small part of the application program, and is executed on target processor. It administrates the power control chip by sending control codes through I/O ports of a target processor.

The power control chip has simple hardware architecture. It does not need to be redesigned for specific target processor,

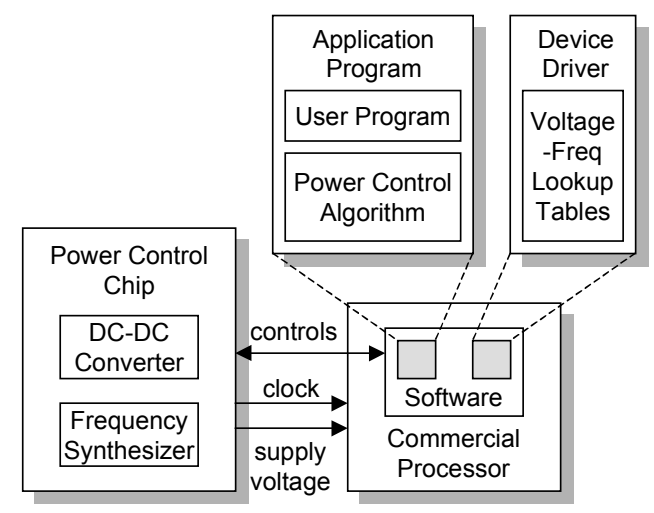

Fig. 1. Proposed run-time power control scheme. because all processor-dependent parameters are stored in a separate device driver program. By using the device driver that can be modified easily when the version of the target processor is changed, the proposed system guarantees the "binary-code compatibility" for any versions of the upwardcompatible processor. On the contrary, in the conventional approaches, the application program should be reprogrammed or recompiled. Moreover, in these cases, "binary-code compatibility" is not guaranteed.

Fig. 2 shows the supply voltages and clock frequencies for the target system. The power control chip generates the variable supply voltage $\mathrm{V}_{\mathrm{VAR}}$ and the variable clock frequency $\mathrm{f}_{\mathrm{VAR}}$ from the master supply voltage $\mathrm{V}_{\mathrm{DD}}$ and master clock frequency $\mathrm{f}_{\mathrm{CLK}}$. Since voltage-frequency relationship of each chip is different, variable supply voltage $\mathrm{V}_{\mathrm{VAR}}$ is applied only to core logic of the target processor that consumes most of the power.

To avoid interface problems, all chips on the target system operate at the same clock frequency $\mathrm{f}_{\mathrm{VAR}}$, except for $\mathrm{I} / \mathrm{O}$ chips. Moreover, $\mathrm{f}_{\mathrm{VAR}}$ has only discrete values of $\mathrm{f}_{\mathrm{CLK}}, \mathrm{f}_{\mathrm{CLK}} / 2, \mathrm{f}_{\mathrm{CLK}} / 3$, ... to simplify the interface scheme. The I/O chips should operate at a constant clock frequency $\mathrm{f}_{\mathrm{CLK}}$ to exchange data with external devices such as CRT, LCD, and RF front-end.

Most real-time applications have some given time intervals in which a certain amount of task should be executed. For example, real-time MPEG-4 SP@L1 video coding should process a picture in $1 / 15$ second. For the rest of this paper, we refer this time interval as a sync frame.

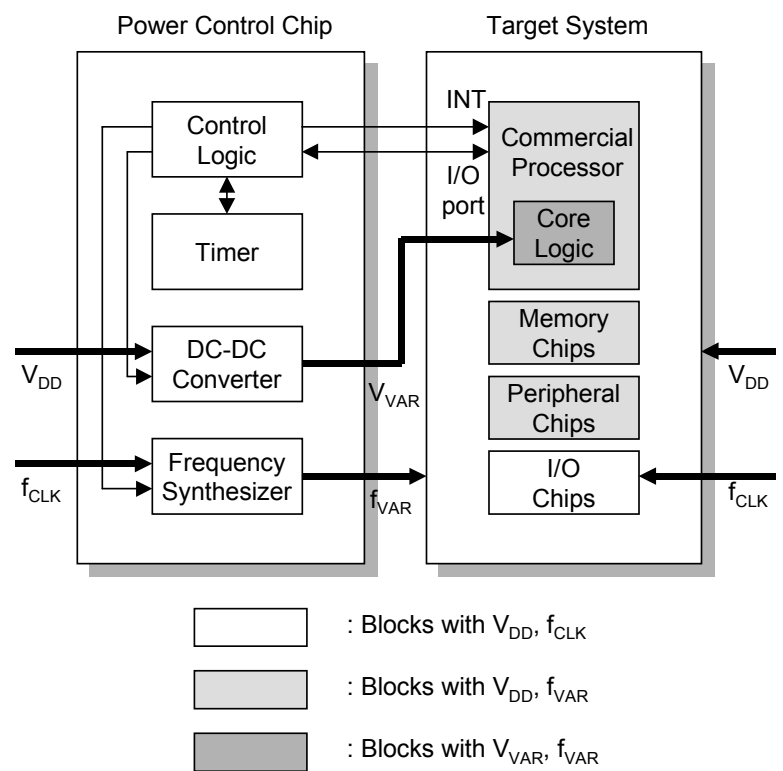

Fig. 2. Supply voltages and clock frequencies for the target system. 
In the proposed power control scheme, every sync frame is divided into several timeslots. For each timeslot, target execution time $T_{\text {TAR }}$ is calculated, and $f_{\mathrm{VAR}}$ is determined to finish the task of a given timeslot within $\mathrm{T}_{\mathrm{TAR}}$ to guarantee the real-time execution. $V_{\mathrm{VAR}}$ is determined from $\mathrm{f}_{\mathrm{VAR}}$, based on the voltage-frequency relationship of the target processor.

The proposed power control algorithm for real-time application is shown in Fig. 3. Application program has two loops: one for the sync frame and the other for the timeslot. The device driver program has two lookup tables: one for voltage-frequency relationship of target processor and the other for transition delay of the power control chip. These lookup tables are made after the measurement of the target processor and the power control chip.

\section{SOFTWARE ALgORITHM AND HARDWARE OPERATION}

\section{A. $f_{\text {VAR_- }} V_{\text {VAR_c }}$ calculate()}

Fig. 4 describes the algorithm how to determine $V_{\mathrm{VAR}}$ and $f_{V A R}$ for each timeslot. The important point is that even for the worst case, all the tasks assigned in a sync frame should be done within that sync frame to achieve the real-time execution. The following algorithm guarantees this point.

(1) For the i-th timeslot, Current time $\mathrm{T}_{\mathrm{Ci}}$ is taken from the internal timer in the power control chip. Target time $\mathrm{T}_{\mathrm{TARi}}$ is calculated as $\mathrm{T}_{\mathrm{TARi}}=\sum \mathrm{T}_{\mathrm{Si}}-\mathrm{T}_{\mathrm{Ci}}$, where $\mathrm{T}_{\mathrm{Si}}$ is execution time limit of the timeslot at maximum clock frequency.

(2) Estimated worst-case execution time $T_{\mathrm{fk}}$ is calculated as $\mathrm{T}_{\mathrm{fk}}=\mathrm{T}_{\mathrm{TD}}+\mathrm{T}_{\mathrm{Si}} \times\left(\mathrm{f}_{\mathrm{CLK}} / \mathrm{f}_{\mathrm{k}}\right)$ if a clock frequency is chosen
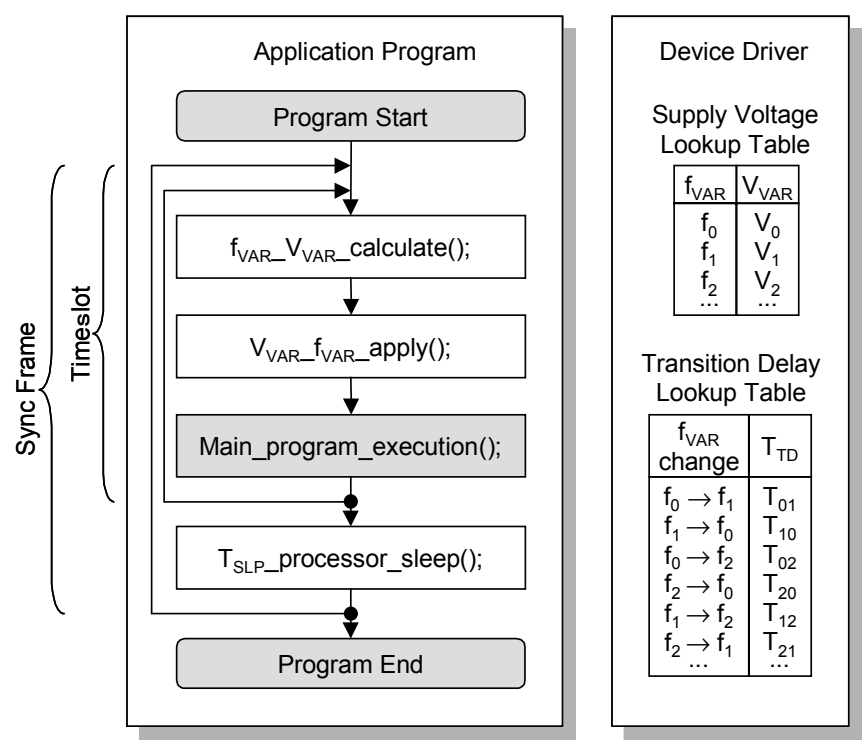

Fig. 3. Application program and embedded power control algorithm.

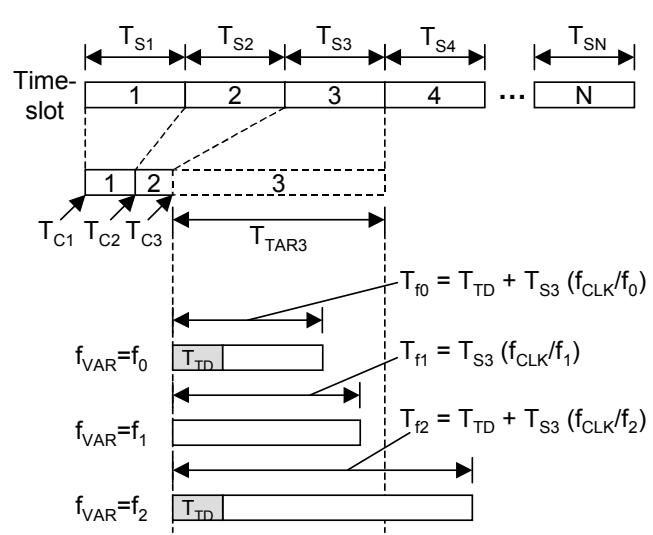

Fig. 4. Determination of $\mathrm{V}_{\mathrm{VAR}}$ and $\mathrm{f}_{\mathrm{VAR}}$.

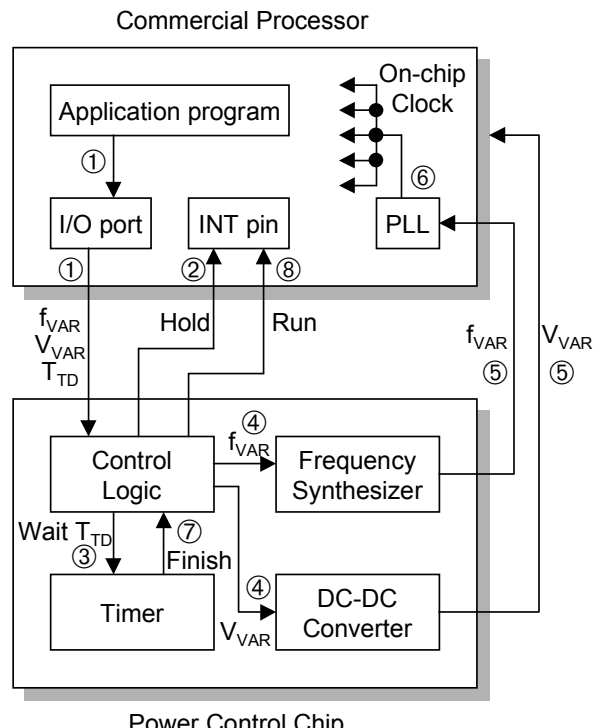

(a) Block diagram.

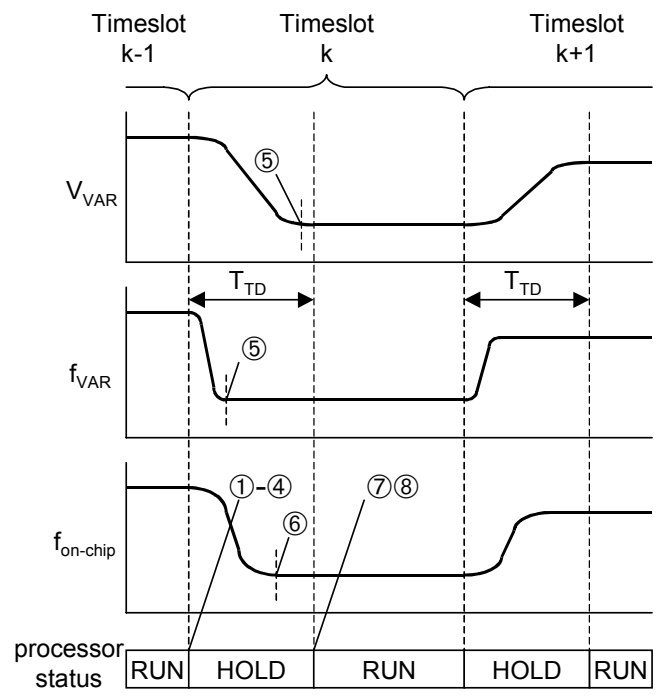

(b) Timing diagram.

Fig. 5. Applying $f_{\mathrm{VAR}}$ and $\mathrm{V}_{\mathrm{VAR}}$ to the target processor. 
as $\mathrm{f}_{\mathrm{k}}(\mathrm{k}=1,2,3 \ldots)$. Note that there is no transition delay if the clock frequency $f_{k}$ does not change from a timeslot to the next timeslot. In Fig. $4, \mathrm{~T}_{\mathrm{fl}}=\mathrm{T}_{\mathrm{S} 3} \times\left(\mathrm{f}_{\mathrm{CLK}} / \mathrm{f}_{1}\right)$ because $\mathrm{f}_{\mathrm{VAR}}=\mathrm{f}_{1}$ for the previous timeslot.

(3) Clock frequency $f_{V A R}$ is determined as the minimum clock frequency $f_{k}$ whose estimated worst-case execution time $\mathrm{T}_{\mathrm{fk}}$ does not exceed the target time $\mathrm{T}_{\mathrm{TAR}}$. Note that real execution time is always smaller than the target time, which guarantees the real-time execution.

(4) Supply voltage $V_{V A R}$ is determined from $f_{V A R}$, using the lookup table in the device driver.

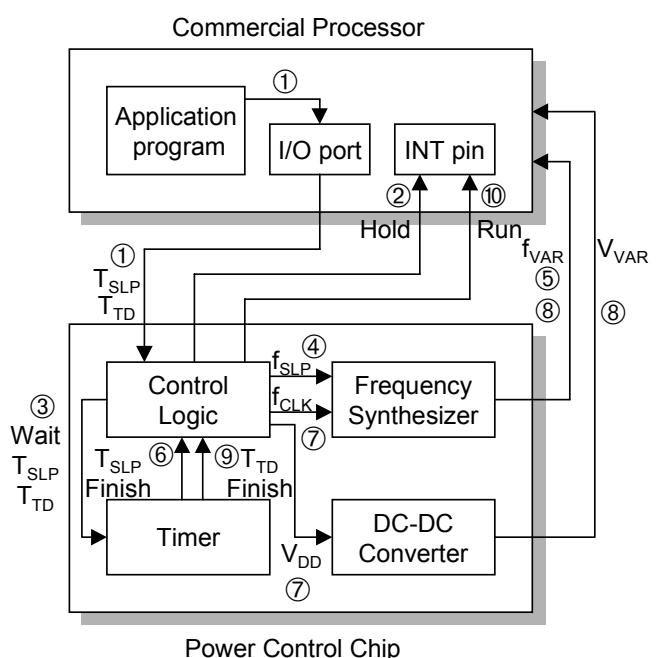

(a) Block diagram.

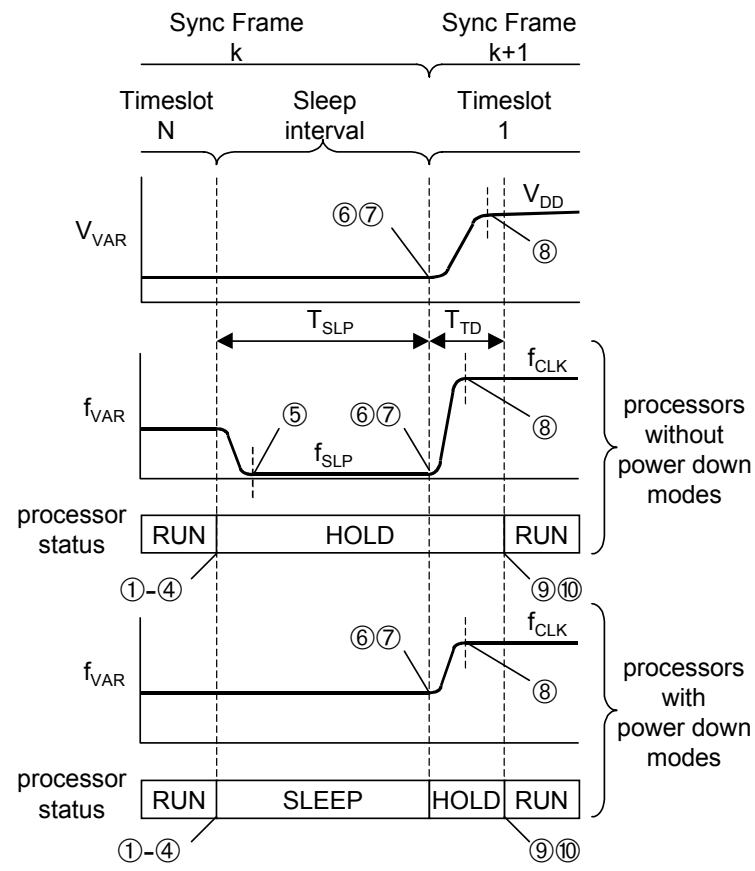

(b) Timing diagram.

Fig. 6. Making the target processor idle at the end of a sync frame.

\section{B. $f_{V A R \_} V_{V A R \_a p p l y}()$}

After $f_{V A R}$ and $V_{V A R}$ are determined in the application program, these values are transferred into the power control chip using I/O instructions as shown in Fig. 5.

During the transition delay $\mathrm{T}_{\mathrm{TD}}$, the clock frequency and the supply voltage are unstable, which may cause invalid operation of the target processor. To avoid this problem, the power control chip makes the target processor in a HOLD state, where the target processor stops running in this interval. The power control chip waits for $\mathrm{T}_{\mathrm{TD}}$ using an internal counter and then issues the interrupt for the target processor to wake up. Without this internal timer in the power control chip, there is no way for the processor to restart.

Many commercial processors have an internal phase-lock loop (PLL) for clock generation. In this case, settling time of an internal PLL should be taken into account in measuring $\mathrm{T}_{\mathrm{TD}}$.

\section{C. $T_{\text {SLP_processor_sleep }()}$}

In real-time applications, there is some idle time at the end of each sync frame, because the real execution time of each sync frame is always smaller than the worst-case execution time. Even in this case, $\mathrm{V}_{\mathrm{VAR}}$ cannot be reduced to zero lest all internal data in the processor should be lost and the interrupt signal should be neglected. The clock frequency, however, can be zero, which results in no power consumption during the idle time. For some processors in a dynamic design style that has the minimum operating frequency, the $f_{V A R}$ should be set equal to the minimum frequency $f_{S L P}$ that the power control chip can provide.

Recently, many commercial processors provide a SLEEP mode for minimum power consumption, where most part of the processor stops running and internal clock goes zero. In this case, we don't need to control the clock frequency. The power control chip makes the target processor in a SLEEP state, instead of a HOLD state. Fig. 6 shows how to make the target processor idle at the end of sync frame.

\section{Performance Evaluation}

In order to evaluate the proposed power control scheme, we applied it to MPEG-4 video encoding, which is one of the typical real-time portable low-power applications for mobile equipments.

We created real-time MPEG-4 SP@L1 video encoding 
software running on Intel Pentium II [7] processor, and measured the execution time of every timeslot and every function module. Effect of operating system (OS) was measured and eliminated, using Intel VTune Performance Analyzer [8] program. Execution cycles of each timeslot were calculated from this timing information.

Power consumption was calculated using Eq. (1), and voltage-frequency relationship was obtained from Eq. (2), based on the alpha-power delay model [9].

$$
\begin{aligned}
& P=\Sigma P_{S L}, \quad P_{S L} \propto V_{S L}^{2} N_{S L} \\
& \frac{1}{f_{S L}} \propto \frac{V_{S L}}{\left(V_{S L}-V_{T}\right)^{\alpha}},
\end{aligned}
$$

where $\mathrm{P}$ is total power consumption, and $\mathrm{P}_{\mathrm{SL}}, \mathrm{V}_{\mathrm{SL}}, \mathrm{N}_{\mathrm{SL}}$, and $\mathrm{f}_{\mathrm{SL}}$ signify power consumption, supply voltage, execution cycles, and clock frequency of a given timeslot, respectively, $\mathrm{V}_{\mathrm{T}}$ is the threshold voltage of the processor, and $\alpha$ is the velocity saturation index.

Simulation conditions are summarized in Table 1. In the modeling of the voltage-frequency relationship, $\mathrm{V}_{\mathrm{DD}}, \mathrm{V}_{\mathrm{T}}$, and $\alpha$ are assumed to be $2.5 \mathrm{~V}, 0.5 \mathrm{~V}$, and 1.3 , respectively, but the normalized power consumption is not sensitive to these values if the parameters are chosen in a practical range.

Fig. 7 shows the power consumption using the proposed power control scheme. This power consumption is normalized by $\mathrm{P}_{\mathrm{FIX}}$, which is the power consumption with a fixed supply voltage and a fixed clock frequency.

The theoretical limit of the power consumption is also calculated using a post-simulation analysis. This theoretical

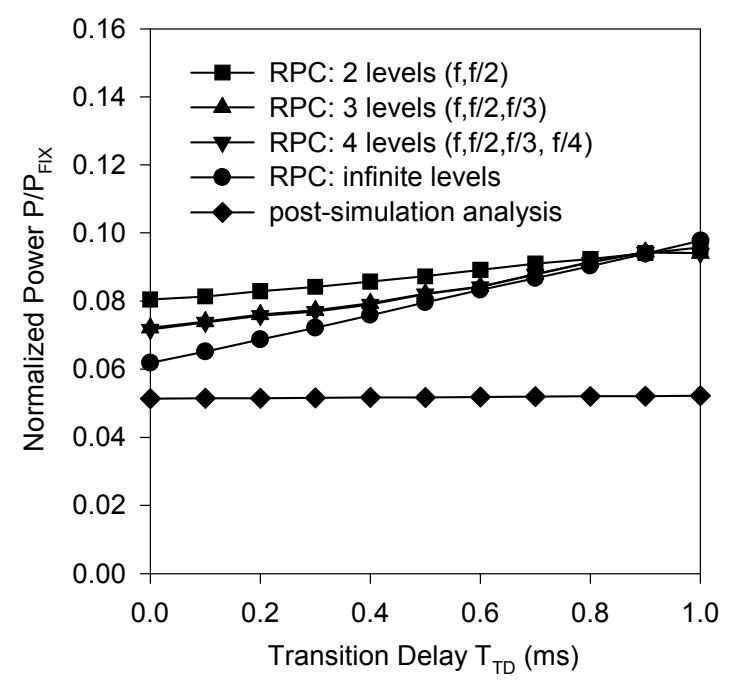

Fig. 7. Normalized power consumption.

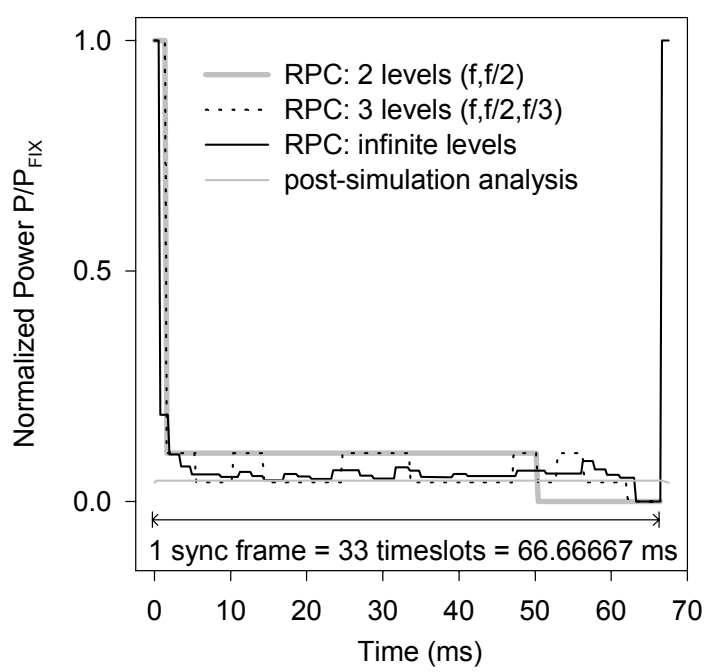

(a) Power.

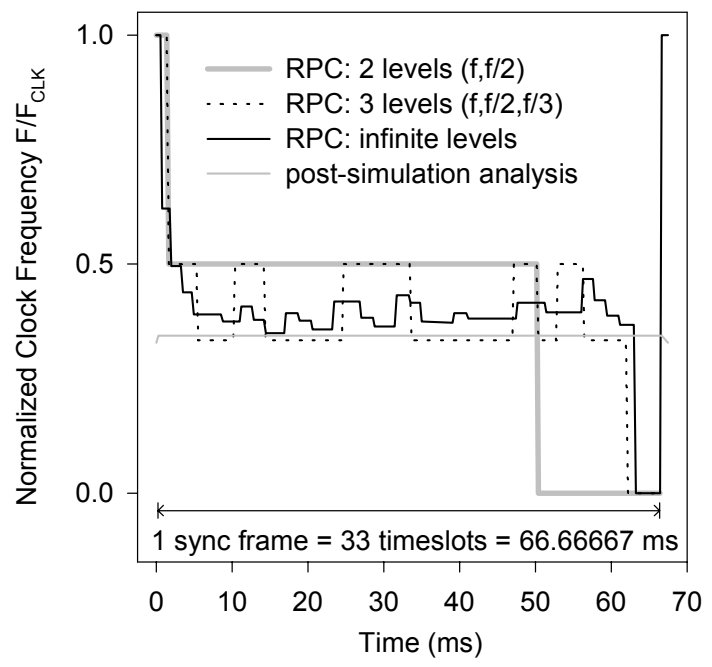

(b) Clock frequency.

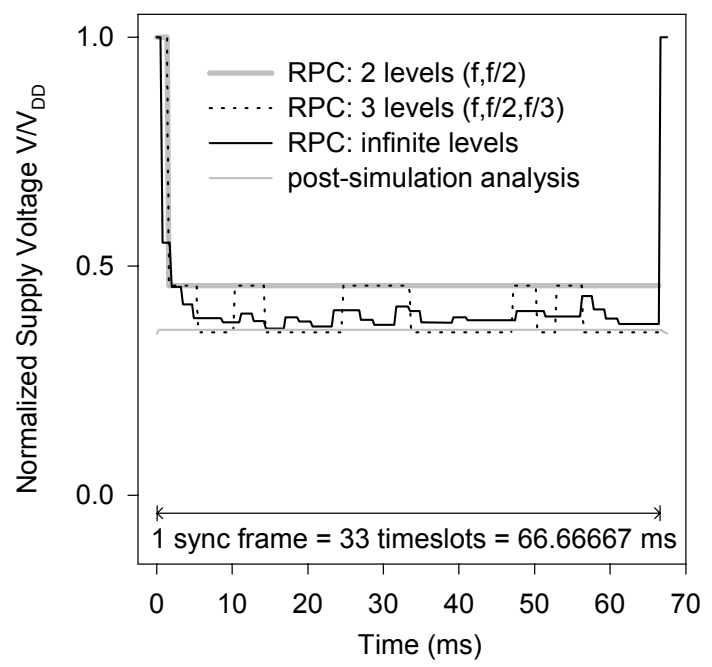

(c) Supply voltage.

Fig. 8. Power, clock frequency, and supply voltage of the 194th sync frame 
limit is optimal in energy saving but can not be realized, because $V_{\text {VAR }}$ and $f_{V A R}$ are determined knowing exact number of cycles required for the sync frame. This can be only achieved by a two-pass process, which is unfeasible in real environments.

From Fig. 7, it is seen that the proposed power control scheme has about 90 94\% power reduction, while the theoretical limit has $95 \%$ power reduction. Also, only two (= $f, f / 2)$ or three $(=f, f / 2, f / 3)$ discrete levels of the clock frequency are sufficient. Power efficiency decreases as the transition delay of the power control chip increases, because the target processor stops its execution during the transition delay.

Fig. 8 shows the normalized transient curves of power, $f_{V A R}$, and $V_{V A R}$ of the 194th sync frame when the transition delay $\mathrm{T}_{\mathrm{TD}}=0.5$ milliseconds.

TABLE I

SiMULATION CONDITIONS

\begin{tabular}{ll}
\hline Target application & MPEG-4 SP@L1 video encoding \\
Real-time algorithm & QCIF (176 pels $\times 144$ pels), 15 Hz \\
Input picture format & 99 \\
Number of macroblocks in a picture & 5 (IPPPP IPPPP IPPPP...) \\
Distance between I-pictures & Full search \\
Method of motion estimation & $\pm 7 \times \pm 7$ \\
Search ranges of motion estimation & \\
Voltage-delay modeling & $2.5 \mathrm{~V}$ \\
External supply voltage & $0.5 \mathrm{~V}$ \\
Threshold voltage & 1.3 \\
$\alpha$ & \\
$\begin{array}{l}\text { Power control algorithm } \\
\text { Length of sync frame }\end{array}$ & $66.67 \mathrm{~ms}$ \\
Length of timeslot & $2.020 \mathrm{~ms}$ \\
Number of timeslots in a sync frame & 33 \\
\hline
\end{tabular}

\section{Conclusion}

A novel run-time power control scheme is proposed using a software feedback loop suited for real-time applications. It employs a power control chip with an on-chip DC-DC converter and a frequency synthesizer, and an embedded runtime power control algorithm using the software feedback loop.

The proposed power control scheme guarantees the realtime operation, and optimizes the supply voltage in run-time while the conventional approaches do in a compile time. It can be directly applicable to many existing processors without hardware redesign, and also has the "binary-code compatibility" for generations of a processor series. It avoids interface problems with external memories, peripheral chips and external devices, by exploiting discrete clock frequency $\mathrm{f}_{\mathrm{CLK}}, \mathrm{f}_{\mathrm{CLK}} / 2, \mathrm{f}_{\mathrm{CLK}} / 3, \ldots$

When applied to real-time MPEG-4 SP@L1 video encoding, the proposed power control scheme is shown to achieve more than $90 \%$ power reduction compared with the fixed frequency and voltage scheme, while guaranteeing the real-time operation. Currently, hardware implementation of the proposed power control scheme is in progress.

\section{ACKNOWLEDGEMENTS}

This research was partly supported by Mirai-Kaitaku Project.

\section{REFERENCES}

[1] A. Chandrakasan, V. Gutnik, and T. Xanthopoulos, "Data driven signal processing: an approach for energy efficient computing," Proceedings of International Symposium on Low Power Electronics and Design (ISLPED'96), pp. 347-352, 1996.

[2] A. Chandrakasan and R. Brodersen, Low Power Digital CMOS Design, Kluwer Academic Publishers, 1995.

[3] T. Ishihara and H. Yasuura, "Power-Pro: programmable power management architecture," Proceedings of Asia and South Pacific Design Automation Conference (ASP-DAC'98), pp. 321-322, 1998.

[4] T. Ishihara and H. Yasuura, "Voltage scheduling problem for dynamically variable voltage processors," Proceedings of International Symposium on Low Power Electronics and Design (ISLPED'98), pp. 197-202, 1998.

[5] T. Pering, T. Burd, and R. Brodersen, "The simulation and evaluation of dynamic voltage scaling algorithms," Proceedings of International Symposium on Low Power Electronics and Design (ISLPED'98), pp. 76-81, 1998.

[6] ISO/IEC JTC1/SC29/WG11 N2202, "Coding of audio-visual objects," May 1998.

[7] http://www.intel.com/PentiumII

[8] http://www.intel.com/vtune/analyzer

[9] T. Sakurai and A. Newton, "Alpha-power law MOSFET model and its application to CMOS inverter delay and other formulas," IEEE Journal of Solid State Circuits, vol. 25, no. 2, pp. 584-594, Apr. 1990. 